\title{
Overview of Human Intelligence Measurements and Future Development Prediction
}

\author{
Shiyu Zuo \\ The University of Manchester, faculty of biology, medicine, and health 518000, China \\ Corresponding author. Email:shiyu.zuo@student.manchester.ac.uk
}

\begin{abstract}
Current intelligence tests rely on performance-based paradigm which possesses flaws that cannot be completely avoided. Based on previous studies, this essay discussed new paradigms that can potentially reduce the flaws including genes prediction, brain-scanning technique, and overexcitability tests. On the contrary, the analysis also included recent findings and theories that may lead to a new understanding towards intelligence. Thus, in this essay, the author suggests short-term and long-term directions for future intelligence tests improvements. In short-term, based on the differences in brain structures of males and females that were found in previous study, it is possible that males and females reach the same level of cognitive abilities with different neural substrates. Thus, it can be necessary that the brain-scanning technique for intelligence tests should develop 2 different paradigms for males and females. In the long-term, we emphasize the importance of Embodied Cognition Theory in testing human intelligence in which stated that it may be too deterministic to estimate human intelligence only by observing brain structures.
\end{abstract}

\section{Keywords: Intelligence test, brain-scanning, genetic prediction, Embodied Cognition, overexcitability}

\section{INTRODUCTION}

Since the invention of the first intelligence test, the Binet-Simon scale, intelligence testing has been studied and improved for more than 100 years. Nowadays, the most widely used intelligence scale is WAIS for both adolescents and adults [1]. The latest version of WAIS is WAIS-IV, it is a composite of four factors (verbal comprehension, perceptual reasoning, working memory, and processing speed) that integrate researches of intelligence, cognitive neuroscience, and ageing [2].

Despite the intelligence tests has been improved for more than 100 years, the essential underlying problems are never solved. Even though the data collection for WAIS-V is progressing through 2020 spring [3], the problems of WAIS series are not likely to be overcome. Current intelligence tests have at least three flaws. Firstly, they are mostly performance-based tests. This means that the performance on the tests are unlikely to represent one's mental ability perfectly because the performances can be influenced by other factors. A classic example is given by Haier [4] in which he stated that although SAT is highly correlated to intelligence test scores [5], the SAT scores of a student can be lowered due to bad health conditions or be raised by SAT preparatory courses but it does not mean that the student's IQ is raised in either circumstance. Secondly, the performance-based tests have low reliability for extreme-IQ subjects because of the ceiling effect and the floor effect. Thirdly, current intelligence tests are performance-based tests that estimate one's theoretical relative rank order in the population. Therefore, it is impossible to eliminate standard error. Because of the disadvantages, it is important to develop a new testing paradigm that is based on mechanism of IQ and generate ratio data or can at least remedy current intelligence tests. This essay will review and discuss the achievements made in genetics and neuroscience and discuss the probability of developing mechanism-based tests in each area. In particular, we will discuss the development prospects of each directions in order to suggest the efficacy and future application scenarios respectively. In consideration of the knowledge gaps, it is hard to develop a decent mechanism-based intelligence test. Thus, we also suggest some findings that can be integrated into current intelligence tests based on the studies on the extreme-IQ population. Additionally, based on some counter-intuitive findings or theories, we also suggest the short-term and long-term aims for future researches that tries to develop new testing paradigms. 


\section{OVERVIEW OF HUMAN INTELLIGENCE MEASUREMENTS}

\subsection{Genetic Prediction:}

Given the disadvantages of current intelligence tests, some studies of genes and neuroscience have some new achievements on developing mechanism-based intelligence tests. One of the most popular directions is to predict IQ with genes. For example, the genome-wide polygenic scores for years of education and intelligence can predict more than $10 \%$ of the variance in intelligence [6]. Moreover, the prediction can be improved through several methods, such as using larger GWA samples and whole-genome sequencing [7] and using samples with extreme high IQ [8]. However, the genetic predictions are unlikely to replace current IQ tests based on existing results. Firstly, according to the twins and adoption studies, DNA difference can only explain $50 \%$ of the variance of intelligence approximately. This means that even the perfect gene-prediction model can only explain half of the variance of intelligence, which makes the prediction not reliable enough. Secondly, the genetic predictions completely ignore the changes of IQ throughout the life. Generally, many studies concluded that IQ is relatively stable throughout life. For example, Deary et al. [9] found that the IQ scores at age 11 and age 77 have a strong positive correlation of 0.73 . However, recent study by Ramsden et al., [10] finds that the verbal IQ $(-20$ to +23$)$, performance IQ $(-18$ to +17$)$, and full-scale IQ $(-18$ to +21$)$ all have the potential to decrease or increase more than 1 SD (15 points). Furthermore, the ratios of the sample show that significant IQ changes are $39 \%$ in VIQ, $21 \%$ in PIQ, and $33 \%$ in FSIQ which suggests that the IQ changes are fairly common in adolescents. This makes the genetic predictions only useful for early warning but not mature intelligence tests.

\subsection{Brain scanning:}

Another method that may replace current intelligence tests is the brain scanning method. Based on the results of the relevant studies, intelligence has been linked to several neural characteristics, including brain volume [11], white matter integrity [12], resting-state brain activity [13] and so on. Until now, it has been shown that it is possible to predict intelligence by observing brain structures. One of the most representative theories is the Parieto-Frontal Integration Theory (P-FIT) [14] which found that the distributions of neural networks can predict intelligence and reasoning performance. In particular, the P-FIT model includes 14 Brodmann Areas that mainly lies in dorsolateral prefrontal cortex, the inferior and superior parietal lobule, the anterior cingulate, and some regions in the temporal and occipital lobes. Jung and Haier also identified some relevant white matter regions in their model. Overall, they believed that the processing stages and interactions of the Parieto-Fronto regions are account for solving abstract questions. Although Jung and Haier admitted that there are probably more regions that play roles in human intelligence, the Parieto-Fronto regions account for the individual differences in intellectual performances.

Given the knowledge of P-FIT, many studies tried to explore the correlations between the functional connectivity of brain and intelligence. A notable example was illustrated by [15]. In this study, they found a significant correlation between fMRI resting-state connectivity, especially between P-FIT regions and matrix reasoning, an estimation of fluid intelligence. The experiment paradigm is shown in Picture 1. As a result, they identified several connections within P-FIT area whose functional connectivity rest-state strength are correlated to intelligence. Another study by Finn et al. [16] tried to predict intelligence with functional connectivity profile of different individuals. They used leave-one-subject-out cross-validation to demonstrate that fluid intelligence can be potentially predicted with connectivity profile of a person. The results showed that the correlation between predicted and observed fluid intelligence of the positive-feature model was $\mathrm{r}=0.50$, $\mathrm{p}<10-9$ for both whole-brain connectivity and frontoparietal networks, demonstrated in Figure 2, The Correlation Between Predicted Intelligence and Observed Intelligence. 

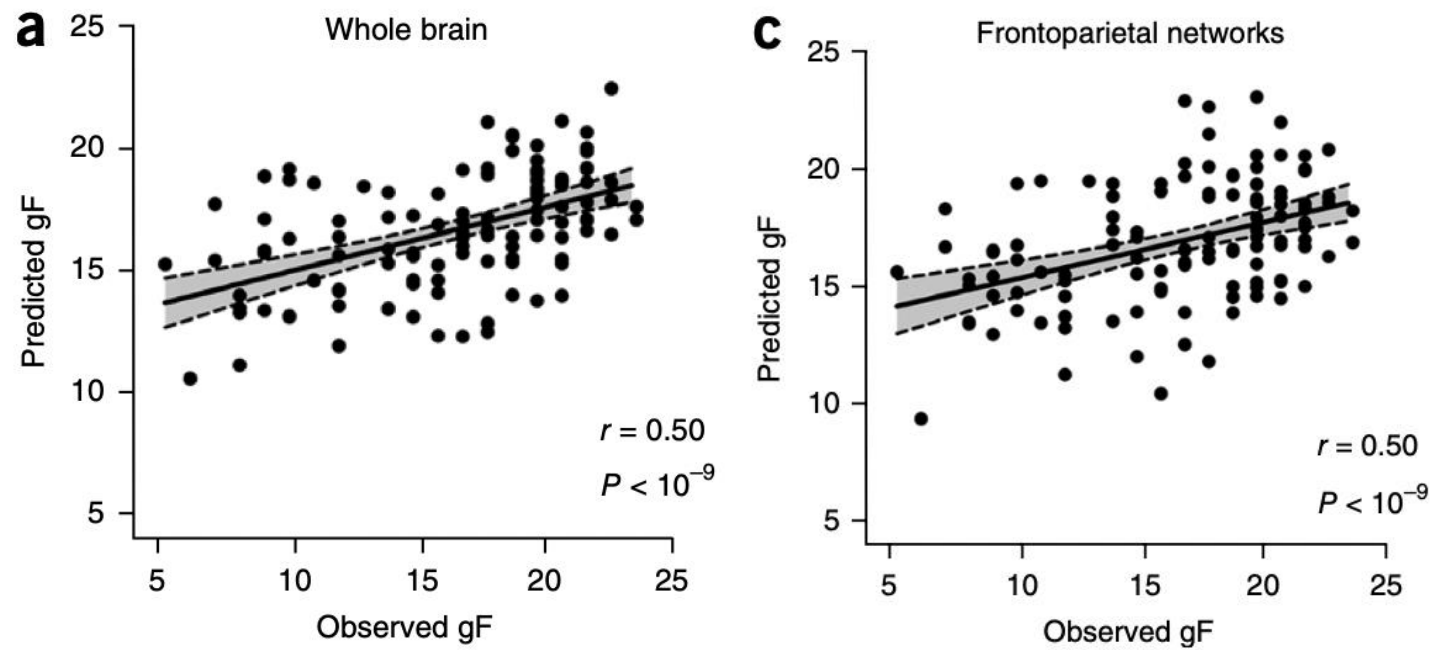

Figure 1 The Correlation Between Predicted Intelligence and Observed Intelligence

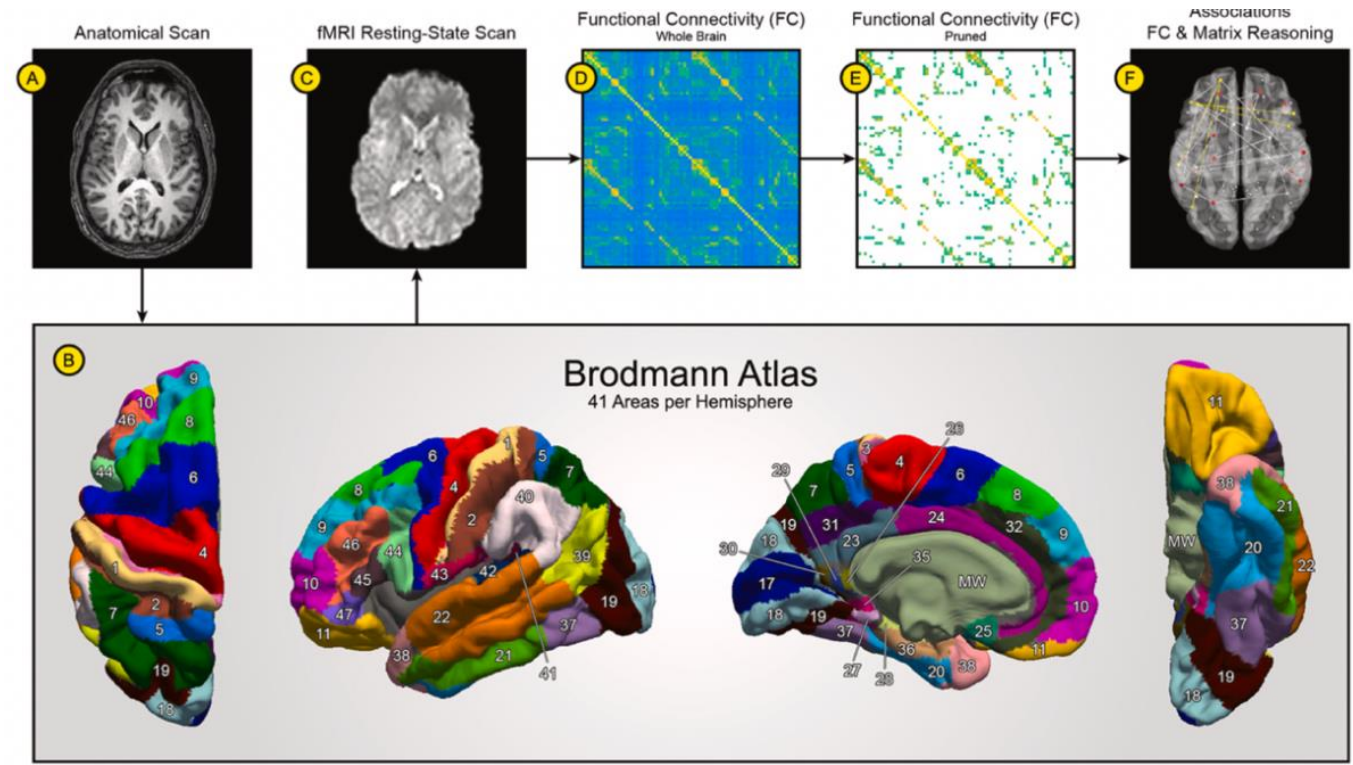

Figure 2 Methodological Sequence for the Parcellation of Brain Scans, The Computation of Resting-State Connectivity Matrices, and the Analysis of Associations Between Functional Connectivity and Matrix Reasoning Performance

\subsection{Overexcitability:}

As previously mentioned, one of the most troublesome problems of current intelligence tests is the high standard error generated when testing high-IQ subjects (145+ IQ). To reduce this problem, a potential method is to integrate other performance-based tests that aim at other characteristics of high-IQ groups when testing their IQ in order to improve the reliability. One important theory about the high-IQ groups is the Overexcitability Theory by Dabrowski [17] which claimed that there are five types of overexcitability that can be frequently found in gifted population including psychomotor, intellectual, emotional, sensual, and imaginational overexcitabilities. According to two meta-analysis, it has been found that the mean scores for all five overexcitabilities are higher in high-IQ groups than in normal groups, especially for the intellectual over [18] [19]. Furthermore, Steenbergen-Hu [19] compared the overexcitability levels and the giftedness for the gifted participants and found that the highly gifted subjects scored significantly higher than their other participants in overexcitability which implied that overexcitability level increases with giftedness and is 
more prominent in highly gifted groups. Based on the results, many researchers believed that it is possible to discriminate high IQ by observing participants' overexcitability levels [20] [21] [22]. Despite the possibility, it is still a far vision and need further studies to make it come true. It is even possible that previous scientists overlooked the correlations between overexcitabilities and intelligence.

\section{FUTURE DEVELOPMENT PREDICTION}

\subsection{Short-term Direction:}

Based on current achievements, the most realistic method that can replace current intelligence tests is brain-scanning method. However, except for keeping study to increase the accuracy, it is possible that different paradigms are needed for different genders to make the brain-scanning method become mature. The possibility is shown in the study by Fraenz et al. [15] in which the model they built explained less variance when the subsample was male compared to the overall samples and did not reach the statistics significance $(\mathrm{R} 2 \mathrm{adj}=2.20 \%, \mathrm{p}=.066)$. On the contrary, the model explained the highest variance in the female subsample $(\mathrm{R}$ adj $=7.23 \%, \mathrm{p}<.001)$. Similar patterns were found in another sample even though the model for the male subsample reached statistics significance $(\mathrm{R} 2 \mathrm{adj}=$ $1.96 \%, \mathrm{p}<.05)$. This finding aligns to previous findings. For example, in the study by Genc et al. [23] , they found that cortex volume predicts the BOMAT scores best in male participants while the functional resting-state connectivity measured based on graph theory predicts best in female participants. Moreover, it has been shown that brain volume is correlated to intelligence [11] but the brains in females are $10 \%$ smaller than that those in males [24] with no substantial difference in IQ. These findings together may suggest that despite similar mental abilities, the mechanisms that females and males rely on can be different. Thus, if future studies are trying to perfect the brain-scanning method, they should aim to develop the tests separately for males and females.

\subsection{Long-term Direction:}

All the methods that have been previously discussed are reductionistic or deterministic to some extent because of the Embodied Cognition Theory in which it stated that the human cognitions are shaped by the whole body and are highly related to perception, action, and emotion [25]. According to this theory, the results of all potential intelligence tests that have been discussed cannot represent the whole human intelligence because neither of them consider the roles perceptions, actions, emotions, and even the whole physical body play in human intelligence. In fact, lots of evidence has indicated this flaw. For example, in recent neuroscience research, it has been found that the neural substrates that support human cognitions are highly linked to those that control sensory and motor [26]. Moreover, previous study found that general intelligence and personality can reliably predict emotional intelligence [27]. More importantly, they found that emotional intelligence and general intelligence share a lot of neural resources especially in frontal, temporal and parietal brain regions, which provided strong evidence for Embodied Cognition Theory. In addition, Kersten [28] revealed that we understand abstract based on internal body-based model. Despite abundant of evidence has revealed the flaws of current intelligence tests, few studies have tried to integrate the findings of Embodied Cognition Theory into intelligence tests. For example, emotional intelligence and general intelligence are often separately measured in psychology researches. However, it is worth questioning that to what extent do these two constructs independent to each other. Moreover, recent studies have also found some correlations between intelligence and perceptual organs. A classic example is the study by Tsukahara and Engle [29] in which they found that larger pupil size is correlated to better cognitive abilities. It is intriguing to conclude that such kind of findings are just byproducts of high intelligence or the products of genes that cause high intelligence. Nevertheless, future study should explore whether there are causal relationships that lies under such findings.

\section{CONCLUSION}

In this essay, we have identified some flaws of current performance-based intelligence and discussed new paradigms that recent studies are developing including genes prediction, brain-scanning techniques, and over excitability tests. In addition, both short-term and long-term improvement directions are also suggested. In near future, researchers should try to develop brain-scanning intelligence tests separately for males and females because of the different mechanisms they rely on to achieve same mental abilities. In far future, Embodied Cognition Theory should be integrated into intelligence tests to reliably test human intelligence. In order to achieve this, the correlations between human cognitions and other abilities, such as perceptions, actions, and emotions should be more deeply studied.

\section{ACKNOWLEDGMENTS}

First, I am deeply indebted to the teachers in Harbour Education and the professors in my university who provided significant supports on my essay writing and knowledge. Further, I gratefully acknowledge the assistance from Professor Melanie Mitchell and her ingenious thesis Why AI Is Harder Than We Think 
which inspired my knowledge about human intelligence in terms of AI. Without all their enlightening instruction and impressive patience, I could not have completed my paper.

\section{REFERENCES}

[1] Hartman DE. Test Review Wechsler Adult Intelligence Scale IV (WAIS IV): Return of the Gold Neuropsychology. 2009;16(1):85-87. doi:10.1080/09084280802644466

[2] Saklofske D.H., Schoenberg M.R. (2011) Wechsler Adult Intelligence Scale (All Versions). In: Kreutzer J.S., DeLuca J., Caplan B. (eds) Encyclopedia of Clinical Neuropsychology. Springer, New York, NY. https://doi.org/10.1007/978-0-387-79948-3_1073

[3] Pearson. Current Opportunities. WAIS 5. Pearson 1996-2020

[4] Haier R. J. (2014). Increased intelligence is a myth (so far). Frontiers in systems neuroscience, 8, 34. https://doi.org/10.3389/fnsys.2014.00034

[5] Frey M. C., Detterman D. K. (2004). Scholastic assessment or $\mathrm{g}$ ? The relationship between the scholastic assessment test and general cognitive ability (vol 15, pg 373, 2004). Psychol. Sci. 15, 641 10.1111/j.0956-7976.2004.00687.x

[6] Allegrini, A. G., Selzam, S., Rimfeld, K., von Stumm, S., Pingault, J. B., \& Plomin, R. (2019). Genomic prediction of cognitive traits in childhood and adolescence. Molecular Psychiatry, 24(6), 819-827.

[7] Wainschtein, P., Jain, D. P., Yengo, L., Zheng, Z., TOPMed Anthropometry Working Group, Trans-Omics for Precision Medicine Consortium, ... Visscher, P. M. (2019). Recovery of trait heritability from whole genome sequence data [preprint]. biorxiv. https://doi.org/10.1101/588020.

[8] Zabaneh, D., Krapohl, E., Gaspar, H. A., Curtis, C., Lee, S. H., Patel, H., ... Breen, G. (2018). A genome-wide association study for extremely high intelligence. Molecular Psychiatry, 23(5), 12261232. https://doi.org/10.1038/mp.2017.121.

[9] Deary, I. J., Whalley, L. J., Lemmon, H., Crawford, J., \& Starr, J. M. (2000). The Stability of Individual Differences in Mental Ability from Childhood to Old Age: Follow-up of the 1932 Scottish Mental Survey. Intelligence, 28(1), 49-55. https://doi.org/10.1016/s0160-2896(99)00031-8

[10] Ramsden, S., Richardson, F. M., Josse, G., Thomas, M. S. C., Ellis, C., Shakeshaft, C.,
Seghier, M. L., \& Price, C. J. (2011). Verbal and non-verbal intelligence changes in the teenage brain. Nature, 479(7371), 113-116. https://doi.org/10.1038/nature10514

[11] Pietschnig, J., Penke, L., Wicherts, J. M., Zeiler, M., \& Voracek, M. (2015). Meta-analysis of associations between human brain volume and intelligence differences: How strong are they and what do they mean? Neuroscience and Biobehavioral Reviews, 57, 411-432.

[12] Ritchie, S. J., Booth, T., Valdes Hernandez, M. D., Corley, J., Maniega, S. M., Gow, A. J., ... Deary, I. J. (2015). Beyond a bigger brain: Multivariable structural brain imaging and intelligence. Intelligence, 51, 47-56.

[13] Avery, E. W., Yoo, K., Rosenberg, M. D., Greene, A. S., Gao, S., Na, D. L., ... Chun, M. M. (2020). Distributed patterns of functional connectivity predict working memory performance in novel healthy and memory-impaired individuals. Journal of Cognitive Neuroscience, 32(2), 241-255.

[14] Jung, R. E., \& Haier, R. J. (2007). The Parieto-Frontal Integration Theory (P-FIT) of intelligence: Converging neuroimaging evidence. Behavioral and Brain Sciences, 30 (2), 135-154.

[15] Fraenz, C., Schlüter, C., Friedrich, P., Jung, R. E., Güntürkün, O., \& Genç, E. (2021). Interindividual differences in matrix reasoning are linked to functional connectivity between brain regions nominated by Parieto-Frontal Integration Theory. Intelligence, $\quad 87, \quad 101545$. https://doi.org/10.1016/j.intell.2021.101545

[16] Finn, E. S., Shen, X., Scheinost, D., Rosenberg, M. D., Huang, J., Chun, M. M., Papademetris, X., \& Constable, R. T. (2015). Functional connectome fingerprinting: identifying individuals using patterns of brain connectivity. Nature Neuroscience, 18(11), 1664-1671. https://doi.org/10.1038/nn.4135

[17] Dabroswki, K. (1967). Personality-shaping through positive disintegration. Boston: Little Brown

[18] Winker D, Voight A (2016) Giftedness and overexcitability: Investigating the relationship using meta-analysis. Gifted Child Quarterly 60: 243-257.

[19] Steenbergen-Hu S (2017) How exactly overexcitability relates to giftedness: A fine-grained look via findings of a new meta-analysis. NAGC Conceptual Foundations Network Newsletter, 44-49. 
[20] Piechowski MM (1979) Developmental potential. In: N Colangelo, RT Zaffrann, New voices in counseling the gifted. (edn), Dubuque, IA: Kendall/Hunt, 25-57.

[21] Ackerman C (1996) Identifying gifted adolescents using personality characteristics: Dabrowski's overexcitabilities. Roeper Review 19: 229-236.

[22] Tucker B, Hafenstein N (1997) Psychological intensities in young gifted children. Gifted child Quarterly 41: 66-75.

[23] Genc, E., Fraenz, C., Schlueter, C., Friedrich, P., Voelkle, M. C., Hossiep, R., \& Guentuerkuen, O. (2019). The neural architecture of general knowledge. European Journal of Personality, 33(5), 589-605.

[24] Ruigrok, A. N., Salimi-Khorshidi, G., Lai, M. C., Baron-Cohen, S., Lombardo, M. V., Tait, R. J., \& Suckling, J. (2014). A meta-analysis of sex differences in human brain structure. Neuroscience and Biobehavioral Reviews, 39, 34-50.

[25] García Rabines, D. (2019). How the body shapes knowledge: Empirical support for embodied cognition de R. Fincher-Kiefer, R. Persona, 022(2), 137-142. https://doi.org/10.26439/persona2019.n022(2).4631

[26] Epstein, R. A., Patai, E. Z., Julian, J. B., \& Spiers, H. J. (2017). The cognitive map in humans: spatial navigation and beyond. Nature Neuroscience, 20(11), 1504-1513. https://doi.org/10.1038/nn.4656

[27] Barbey, A. K., Colom, R., \& Grafman, J. (2012). Distributed neural system for emotional intelligence revealed by lesion mapping. Social Cognitive and Affective Neuroscience, 9(3), 265272. https://doi.org/10.1093/scan/nss 124

[28] Kersten, A. W. (2006). Grounding cognition: the role of perception and action in memory, language, and thinking. D. Pecher, \& R. A. Zwaan (Eds.). Cambridge University Press, Cambridge, 2005. No. of pages 326. ISBN 0-521-83464-3. Applied Cognitive Psychology, 20(2), 275-277. https://doi.org/10.1002/acp.1193

[29] Tsukahara, J. S., \& Engle, R. W. (2021). Is baseline pupil size related to cognitive ability? Yes (under proper lighting conditions). Cognition, 211, 104643.

https://doi.org/10.1016/j.cognition.2021.104643 